\title{
A Study on the Promoting Effect of Augmented Reality Design on Senior High School Students' Understanding of Portrait Depiction in "A Dream of Red Mansions"
}

\author{
Shizhen Zhou ${ }^{1}$ Baojun Chu, ${ }^{1, *}$ \\ ${ }^{1}$ Xiamen Academy of Arts and Design Fu Zhou University, Xiamen, Fujian 361000, China \\ *Corresponding author. Email: t17075@fzu.edu.cn
}

\begin{abstract}
"A Dream of Red Mansions", the peak of Chinese classical literature, reflects the truth and beauty of history and has a strong cultural radiating power and appeal. "Inquiry into the Reading Teaching of the Whole Book" shows that the study of "A Dream of Red Mansions" is conducive to improving the language construction and thinking development of senior high school students. According to John Sweller's cognitive load theory, in the process of knowledge learning, human beings have understanding load in the cognitive system due to individual experience, the essential characteristics of learning materials and the way of material presentation. This phenomenon also acts on the interpretation of "A Dream of Red Mansions" by high school students, resulting in cognitive load. Therefore, on the premise of not affecting the reading habits of traditional novels, this study will use the augmented reality technology to design the portrait depiction text to be intuitive and visible, and to blend the false and the real, so as to make up for the cognitive load of students when interpreting "A Dream of Red Mansions". The intelligent interaction characteristics of augmented reality technology are used to enhance students' audio-visual experience, reduce the cognitive load of describing the intention and emotion of the novel text, so as to deepen students' understanding of classical literature and promote the inheritance and development of Chinese traditional culture.
\end{abstract}

Keywords: Cognitive load, Augmented reality design, Classic, "A Dream of Red Mansions".

\section{INTRODUCTION}

Reading "A Dream of Red Mansions" is helpful to improve students' literary accomplishment, but its text content is complicated, which brings students a certain degree of cognitive load. This research will combine the augmented reality technology and take the portrait depiction in the article as the starting point to bring students an interactive and audio-visual reading experience, reduce cognitive load and deepen students' cognition of classical literature.

\section{UNDERSTANDING LOAD OF TEXTUAL READING}

Chinese classical literature is the essence of Chinese traditional culture, and as the peak of classical literature, "A Dream of Red Mansions" reflects the powerful cultural radiation and appeal through the beauty of words, which has a high research value.

The Chinese Curriculum Standards for General Senior High School (2017 Edition 2020 Revision) clearly states that in order to further improve the comprehensive Chinese literacy of senior high school students and enhance the ability of imagebased thinking, "whole book reading and discussion" is set as the first task group of Chinese learning in senior high school, and is included in the curriculum system. Wang Chunjing [1], Yang Huaiyi [2] and other scholars' "Whole Book Reading Teaching" show that reading "A Dream of Red Mansions" for senior high school students is conducive to language accumulation and construction, thinking development and promotion, 
and cultural inheritance and understanding. Therefore, it is very important to read the text of "A Dream of Red Mansions".

According to John Sweller's cognitive load theory [3], cognitive load includes intrinsic load and extraneous load. Intrinsic cognitive load is determined by the essential content of learning materials and generated in the process of learning interaction, which is positively correlated with the individual's cognitive schema and learning level. The influencing factors of extrinsic cognitive load are the presentation of materials or the design of instruction. In the process of learning or reading, human beings have a load in their cognitive system due to the individual knowledge framework and the form of learning materials.

The great artistic achievement of "A Dream of Red Mansions" is highlighted in the creation of groups of personalized characters with flesh and blood, which reveals the true face of the feudal system and society. Therefore, it is of great significance to study the text of portrait depiction in "A Dream of Red Mansions". However, when high school students read "A Dream of Red Mansions", there is a cognitive load on the portrayal of portrait text. Students with weak thinking ability cannot even conceive a character image model in their minds. A technique is needed to make up for this defect and reduce high school students' cognitive load, which will increase their interest in reading and deepen their understanding and cognition of the classic novel "A Dream of Red Mansions".

\section{AUGMENTED REALITY AND CURRENT STATE OF APPLICATION}

Augumented reality (which is abbreviated as AR) technology is also called mixed reality technology, which is the compound content produced by artificial environment combined with reality environment by computers. The users can see not only the content in reality world through screens, but also the virtual trends information after overlying, which is the enhanced complement of reality situations [4]. The core features are real-time interaction, virtual and reality interaction and threedimensional registration.

Based on the statistical analysis of Apple App Store and Android App Market, this study concludes that AR mostly involves tools, painting and entertainment fields, such as AR ranging, AR constellation and AR audio picture books. Through the analysis of the publication and sales of AR books on Taobao, Dangdang and JD book sales platforms, it is concluded that the application of AR technology in the digital presentation of books is mainly to assist children's cognition (such as children's picture books and other early education books). There are not many researches on the integration of $\mathrm{AR}$ and text novels at the present stage, so this study focuses on the integration of AR technology into the image thinking supplement of the portrait depiction in the novel "A Dream of Red Mansions".

\section{STRENGTHENING THE ADVANTAGES OF REALISTIC APPLICATION FOR TEXTUAL NOVELS}

First of all, traditional paper books, as a constantly developing information media, have a profound historical accumulation and cultural deposits. Paper books as a carrier of information, readers can be fully engaged in the reading process, page by page to stimulate the reader's desire, trigger real experience and emotional communication. Augmented reality's display of the characters' portraits in "A Dream of Red Mansions" has shaped the cognitive process of readers, promoted people's cognitive investment and improved individual cognition. The combination of AR technology and novel text not only retains the texture of traditional print media reading and browsing, but also enriches the expression form of book content by superimposition of virtual objects. Through immersive interactive experience, it stimulates reading interest and promotes emotional investment of readers.

Secondly, when high school students read "A Dream of Red Mansions", they will generate strong cognitive load due to the abstract form of the book text, their own cognitive model and the resistance of knowledge frame formed after reading the Red Mansions. Compared with traditional books, integrating AR technology into paper books can transform complex indirect experience into vivid direct experience, three-dimensional and dynamic processing of abstract content, and make virtual reading experience and technology sense to a higher level. It reduces the cognitive difficulty of portrait depiction in the text of "A Dream of Red Mansions", and greatly reduces the cognitive load generated by high school students in reading, so as to deepen the interpretation of "A Dream of Red 
Mansions" and the understanding of classical literature.

Thirdly, the reading of traditional text books is a cognitive mode of unilateral transmission, which relies on the reader's own image thinking to interpret the text content, and requires readers to mobilize their imagination to fill in the abstract book content, while people with weak image thinking ability will have some difficulties in text interpretation and cognition. The injection of $\mathrm{AR}$ technology makes the knowledge transmission in "A Dream of Red Mansions", especially the portrait depiction, more in line with the needs of humanized development, enhances the auditory and visual senses, and turns it into a "surreal" sensory transformation, that is, "the static picture in reality appears on the paper" [5], thus enjoying the twoway dynamic reading experience. Interactive and multi-dimensional immersive reading enriches the form of expression of book content, not only surpasses the boundary of book form, but also breaks the barrier of time and space [6], reshaping the form of media and the pattern of knowledge dissemination.

Finally, the combination of "A Dream of Red Mansions" and AR technology reconstructs the form of reading. The knowledge dissemination and cultural inheritance of the traditional book "Writings are for conveying truth" has been changed by the augmented reality technology. In a nutshell, AR technology breaks down the boundaries between the real and the fake, and elevates the reading experience to a whole new level.

\section{DESIGN IMPLICATION}

The ultimate goal of the design is to write an augmented reality application on the mobile terminal, scan the code through the AR application, display the 3D model corresponding to the specific text paragraph, and clarify the intention emotion expressed by the text content.

Design idea: carefully read the text of "A Dream of Red Mansions", select the typical portrait depiction paragraphs in the book, and analyze and summarize the agreed conclusion through visiting redologists and litterateurs. Collect relevant film and television works and picture book illustrations for analysis and research, design and draw the plan, confirm the final plan after a series of optimization and improvement, build a three-dimensional model and map according to the drawing pattern, and then focus on the improvement of details after getting the rendering effect, cycle iteration. Finally, the integrated development of AR display program is completed, and the functions of rotation, zoom and screenshot are realized. The design idea is shown in "Figure 1".

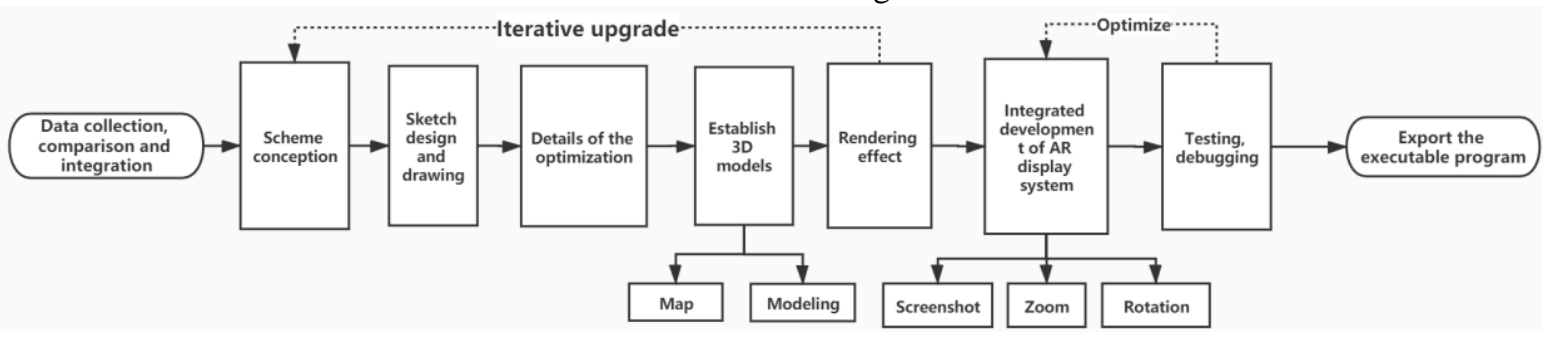

Figure 1 Design idea.

\subsection{Searching the Original Text of the Book and Comparing the Research of the Data}

The following is a portrait of Wang Xifeng when he first met Lin Daiyu:

Her chignon was enclosed in a circlet of gold filigree and clustered pearls. It was fastened with a pin embellished with a fying phoenixes, from whose beaks pearls were suspended On tiny chains. Her necklet was of red gold in the form of a coiling dragon. Her dress had a fitted bodice and was made of dark red silk damask with a pattern of flowers and butterflies in raised gold thread. Her jacket was lined with ermine. It was of a slate-blue stuff with woven insets in coloured silks. Her underskirt was of a turquoise-coloured imported silk crêpe embroidered with flowers [7].

Comparison of film and television works and picture book materials, as shown in "Figure 2": 


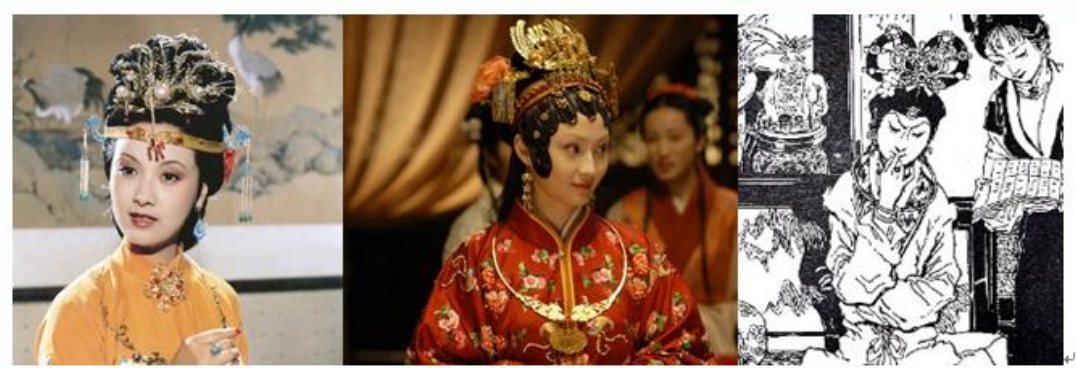

Figure 2 The reappearance of the image of Wang Xifeng by film and television and picture books.

The following is a portrait of Jia Baoyu when he appeared on stage when Jia Baoyu and Lin Daiyu first met:

His side hair was dressed in a number of small braids plaited with red silk, which were drawn round to join the long hair at the back in a single large queue of glistening jet black, fastened at intervals from the nape downwards with four enormous pearls and ending in a jewelled gold clasp. He had changed his robe and jacket for a rather more worn-looking rose-coloured gown, sprigged with flowers. He wore the gold torque and his jade as before, and she observed that the collection of objects round his neck had been further augmented by a padlock-shaped amulet and a lucky charm [7].

Comparison of film and television works and picture book materials, as shown in "Figure 3":



Figure 3 The reappearance of the image of Jia Baoyu by film and television and picture books.

\subsection{Drawing the Final Plan According to the Original Novel}

Through comprehensive analysis, the elements of Wang Xifeng's image mainly include: eight treasures in gold silk with beads, red gold plate with dragon wreath, red phoenix eye and willow leaf eyebrow. The final scheme is shown in "Figure 4":
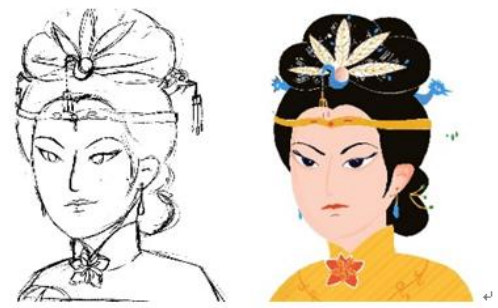

Figure 4 The ultimate scheme of Wang Xifeng.
Through comprehensive analysis, the elements of Jia Baoyu's image mainly include: eight gold treasures pendant horns, collar, and silver-red flowered semi-old big coat. The final plan is shown in "Figure 5":
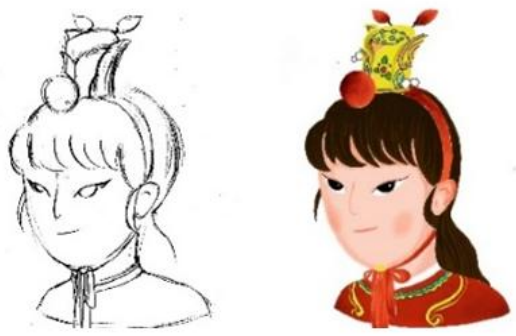

Figure 5 The ultimate scheme of Jia Baoyu.

The design practice development will use Unity3D platform as the main development tool, and combined with Vuforia Engine to assist the implementation. Unity3D is a fully integrated 
professional game engine, which can realize the development of 3D games, architectural visualization, augmented reality, virtual reality and other interactive content, and has the characteristics of cross-platform and multi-compatibility. Vuforia Engine is an augmented reality SDK developed by
Qualcomm, which is now built into Unity3D and can provide multiple recognition methods (image target recognition, multi-target recognition, and column target recognition) with good effect and strong stability. Its workflow is shown in "Figure 6":

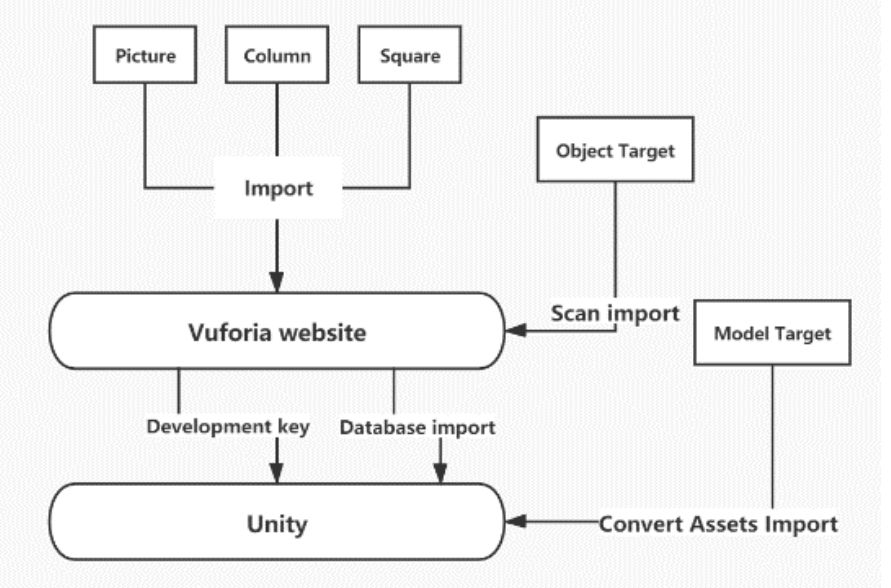

Figure 6 The working process of Viforia.

- Performing preparatory work, including registering Vuforia account and obtaining License Key.

- Transfering the development environment to Unity3D platform and import the key of development license; Set the parameters of the configuration file VUFORIACON figuration; Add and import Database, namely Vuforia identity data, divided into local (device) identity and cloud (cloud) identity. Then add the recognition object. This time, the image Code is used as the recognition object of AR scan. Finally, use $\mathrm{C \#}$ language to write the interactive processing logic.

- Exporting the program to Android or IOS mobile terminal through Unity3D platform, and debug it to see the actual test effect.

The final run usage flow is shown in "Figure 7":

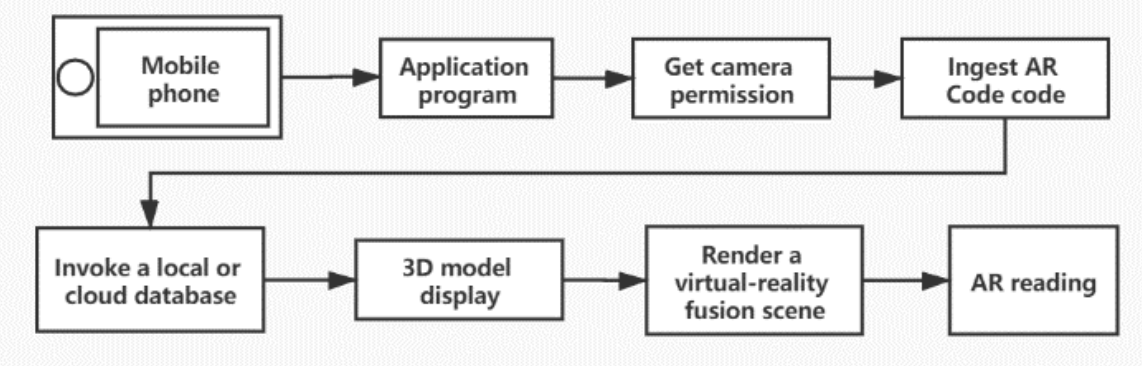

Figure 7 The working process.

\section{CONCLUSION}

Based on the cognitive load theory, this study combines AR technology with the classical novel "A Dream of Red Mansions", focusing on the visual design of portrait text depiction, making reading more in-depth and intuitive, improving the effect of text information and knowledge transmission, and deepening the understanding of
"A Dream of Red Mansions" by high school students.

The combination of AR technology and classical works is a brand new design of user reading experience. The next step will be to extend the research object to the depiction of other things in the classics, such as the depiction of architecture and food. In addition, AR technology will be gradually integrated with the other four classic 
novels. While showing the charm of the four classic novels to the Chinese people, it will enter the foreign book market, carry forward the Chinese culture and enhance the cultural identity.

However, China's AR technology is still in the development stage, and the setting of using mobile devices to view three-dimensional images in the process of reading books also violates users' reading habits. Therefore, the development of AR glasses and the control of the price are imperative. It can be seen that the AR model of reading classical novels still has huge development and application space in the future.

\section{AUTHORS’ CONTRIBUTIONS}

Shizhen Zhou was responsible for writing the manuscript and drawing pictures, and Baojun Chu was responsible for reviewing papers and proposing amendments.

\section{REFERENCES}

[1] Wang Chunjin, Research about the thoughts and practice on the teaching and reading the whole book-Take the reading teaching in A dream in red mansions as examples. J. Chinese teaching in senior middle school,

2017 (10): Page 24-26.

[2] Yang Huaiyi, A probe into the teaching of reading the whole book in senior high schoolTake the portrait text of the novel A dream in red mansions for example. J. modern middle and primary school education, 2019(3): Page 29-32.

[3] John Sweller, Paul Ayres, Slave Kalyuga. Gognitive Load Theory M. Springer, New York, NY: January 1st, 2011.

[4] Wang Xuan, Li Lei. On the basis of strengthening realistic children to learn Chinese books designing and develop,emt.J Chinese editor, 2020(08): Page 64-69.

[5] Xue Xuan, Piao Meishan, the analysis of a new type of virtual reading - on the basis of the research on AR technology in the ways of reading. J. Wide angle publishment, 2020(02): Page 47-49.. Nicole, "Title of paper with only first word capitalized," J. Name Stand. Abbrev., in press.
[6] Wang Xiuli, Hu Yukang, the new form of designing for children's books under the technology of AR. Research on publication, 2016(05): Page 48-50.

[7] Cao Xueqin, Gao E in Qing Dynasty wrote A dream in red mansions, while Yang Xianyi, Dai Naiyi translated, M. Beijing, Foreign language publication in January of 2015. 